\title{
Duchenne Muscular Dystrophy: a look at ventilatory disorders
}

\author{
Beatriz Sordi Chara ${ }^{1}$, Tiago Neves Veras ${ }^{2}$, Anna Paranhos Miranda Covaleski ${ }^{3}$, and \\ Marcus Magno Gonçalves ${ }^{2}$ \\ ${ }^{1}$ Universidade da Região de Joinville \\ ${ }^{2}$ Universidade da Região de Joinville Departamento de Medicina \\ ${ }^{3}$ Universidade Federal de Pernambuco
}

October 5, 2020

\begin{abstract}
Duchenne Muscular Dystrophy is an X-linked inherited disorder and the most common fatal degenerative muscle disease in pediatric patients. Its first symptoms usually manifest at an early age, with weakness, psychomotor developmental delay, evolving to cardiac and ventilatory muscle impairment. The involvement of the pulmonary musculature is demonstrated by the frequent episodes of obstruction and aspirations, sleep-disordered breathing, and by the development of a chronic restrictive breathing pattern. Failure of respiratory mechanics is a major cause of mortality in Duchenne Muscular Dystrophy, and the beginning of lung function deterioration is not always accompanied by symptoms, which reinforces the need for serial evaluations for early initiation of a ventilatory support. The present article is thought to provide a synthesized overview of the assessment and management of Duchenne Muscular Dystrophy ventilatory disturbances.
\end{abstract}

\section{Duchenne Muscular Dystrophy: a look at ventilatory disorders}

Beatriz Sordi Chara ${ }^{1}$;Tiago Neves Veras ${ }^{2}$; Anna Paula Paranhos Miranda Covaleski ${ }^{3}$, Marcus Vinícius Magno Gonçalves ${ }^{4}$

1- Medical student - Department of Medicine, Universidade da Região de Joinville (UNIVILLE), Address: 10 Paulo Malschitzki St, Joinville, SC, 89219710, Brazil;

2 - Medical Doctor, MSc. and Professor of Pediatrics, Universidade da Região de Joinville (UNIVILLE), Address: 10 Paulo Malschitzki St, Joinville, SC, 89219710, Brazil;

3- Medical Doctor, MSc. and Professor of Neuropsychiatry, Universidade Federal de Pernambuco (UFPE), Address: 1235 Professor Moraes Rego Ave, Recife, PE, 5060901, Brazil;

4- Medical Doctor, Ph.D., and Professor of Neurology, Universidade da Região de Joinville (UNIVILLE), Address: 10 Paulo Malschitzki St, Joinville, SC, 89219710, Brazil.

\section{Corresponding Author}

Marcus Vinícius Magno Gonçalves

Department of Medicine - University of the Region of Joinville. Address: 10 Paulo Malschitzki St, Joinville, SC, 89219710, Brazil. Phone number: +55 479 9974-9668. E-mail:mvmpesquisa@gmail.com

\section{Study funding}

No targeted funding reported.

\section{Disclosure}


The authors report no disclosures. All authors and contributors agree to the conditions outlined in the Authorship and Contributorship section of the Information for Authors. The authors have read the Journal's position on issues involved in ethical publication. There was no sponsorship for the scientific article and there was no conflict of interest with all the authors.

\title{
Keywords
}

Duchenne Muscular Dystrophy, Dystrophin, Respiratory Failure, Ventilatory Depression

\author{
ABBREVIATIONS \\ DMD - Duchenne Muscular Dystrophy \\ FVC - Forced Vital Capacity \\ PSG - Polysomnography \\ PEF- Peak Expiratory Flow
}

\begin{abstract}
Duchenne Muscular Dystrophy is an X-linked inherited disorder and the most common fatal degenerative muscle disease in pediatric patients. Its first symptoms usually manifest at an early age, with weakness, psychomotor developmental delay, evolving to cardiac and ventilatory muscle impairment. The involvement of the pulmonary musculature is demonstrated by the frequent episodes of obstruction and aspirations, sleepdisordered breathing, and by the development of a chronic restrictive breathing pattern. Failure of respiratory mechanics is a major cause of mortality in Duchenne Muscular Dystrophy, and the beginning of lung function deterioration is not always accompanied by symptoms, which reinforces the need for serial evaluations for early initiation of a ventilatory support. The present article is thought to provide a synthesized overview of the assessment and management of Duchenne Muscular Dystrophy ventilatory disturbances.
\end{abstract}

\section{HIGHLIGHTS}

- Loss of ventilatory function is one of the most severe complications of DMD;

- Frequent spirometries should be performed since the child is 5 years old

- A reduction in FVC and PEF can be detected before the first motor manifestations;

- One of the main life-threatening conditions related to DMD is sleep-disordered breathing

- Early initiation of patient monitoring, NIV and treatment with steroids are essential.

\section{EDUCATIONAL AIMS}

- Demonstrate the main ventilatory complications of Duchenne Muscular Dystrophy (DMD) and its mechanisms;

- Reinforce the importance of early-onset follow-up and serial assessments to monitor the evolution of DMD ventilatory manifestations;

- Point out the methods of diagnosis and monitoring of ventilatory syndromes associated with DMD;

- Expose available therapies to prevent disease progression.

\section{FUTURE RESEARCH DIRECTIONS}

Future studies may address the limitations that lead to the late diagnosis of DMD and consequently delay the beginning of ventilatory support and prophylactic therapies. It would also be relevant to analyze the effectiveness of the newest pharmacological treatments, in relation to its ability to contain the progression of ventilatory muscle deterioration.

\section{INTRODUCTION:}

Duchenne Muscular Dystrophy is an X-linked inherited disorder and the most common fatal muscle disease in pediatric patients, affecting one of 3,000 - 6,000 male births. It is caused by a mutated encoding gene that produces a dysfunctional dystrophin protein, resulting in progressive degeneration of cardiac and striated 
muscles. DMD diagnosis is sustained by family history, typical clinical presentation, and altered laboratory findings, such as elevated transaminases and creatine kinase, however, is only confirmed through the absence of dystrophin in immunohistochemical tests of muscle biopsy or in genetic tests by detecting mutations in the DMD gene ${ }^{\mathbf{1 , 2}}$. The first symptoms usually manifest at an early age, with weakness of lower limbs and shoulders, psychomotor developmental delay, calf hypertrophy, and, later, with signs of cardiac and ventilatory muscle impairment

$2,3,4,5$.

Loss of respiratory function is one of the most severe complications of DMD. The involvement of chest wall and diaphragm muscles come out as muscle fatigue and thoracic deformities demonstrated by a decreasing Forced Vital Capacity (FVC) ${ }^{\mathbf{1 , 6}}$. Spirometry is one of the leading tools for monitoring DMD, it is widely used and easily accessible. The progressive decline in its parameters, especially FVC and Peak Expiratory Flow (PEF), is an important indicator of clinical deterioration ${ }^{7}$.

Early diagnosis is able to prevent unfavorable outcomes at very young ages. Unfortunately, delays in identifying DMD are common and can be devastating for patients and their families. A study by Wong et al (2015) found a time difference of 6 months to 4 years between the first concerns about the child's development and confirmation of $\mathrm{DMD}^{\mathbf{8}, 9}$. Late diagnoses can impair access to corticosteroids, first-line drugs capable of altering the natural history of the disease ${ }^{\mathbf{9}}$.

The purpose of this non-systematic review is evaluate the main aspects of recognition, monitoring, and management of pulmonary changes in DMD.

\section{METHODS}

We performed searches for articles in multiple health-related databases, such as Pubmed, Scielo and Scholar Google, with a time restriction of 5 years, using the keywords "Duchenne Muscular Dystrophy", "ventilatory failure", "lung function", emphasizing in case series, cross-sectional studies, case-control, cohorts, clinical trials, systematic and non-systematic reviews.

\section{DISCUSSION}

Failure of ventilatory mechanics is a major cause of mortality in DMD. A study conducted by McDonald and colleagues (2016) with 114 children, detected important pulmonary complications in $34 \%$ of them. It is estimated that 55-90\% of DMD's patients die from respiratory insufficiency between 16.2 and 19 years of age ${ }^{\mathbf{1 0}}$. The spectrum of symptoms triggered by the involvement of the pulmonary musculature ranges from frequent obstructions, due to the weakening of upper airway muscles, to a chronic restrictive breathing pattern, as a result of chest-wall muscles degeneration. If spinal muscles are affected, orthopedic abnormalities may be present, such as scoliosis. These alterations, when associated with difficulty in expectorating and coughing, can lead to chronic aspirations, atelectasis, and fibrosis ${ }^{\mathbf{1 1 , 1 2}}$.

One of the main life-threatening conditions related to DMD is sleep-disordered breathing, especially nocturnal hypoventilation, an independent predictor of severity, and a marker of ventilatory failure onset. It is more prevalent in the Rapid Eye Movement stage of sleep and can be identified by Polysomnography (PSG) with transcutaneous $\mathrm{CO} 2$ capnography ${ }^{\mathbf{3}}$. In a 5 -year retrospective analysis performed with 34 male patients with DMD, Hoque et al (2016) observed that 15 of them didn't present any abnormalities in PSG, 10 had obstructive sleep apnea, and 7 developed hypoventilation ${ }^{\mathbf{1 3}}$. Beyond PSG studies, it is important to continuously monitor the pulmonary function of DMD patients, assessing forced vital capacity, ventilatory muscle strength, and oximetry, in order to recognize signs of clinical worsening like daytime hypoventilation 12 .

During the ambulatory stage, frequent spirometries should be performed since the child is 5 years old. Interestingly, the Forced Vital Capacity curve increases for a certain period, following the patient's growth, until it reaches a plateau and begins to decline. It is important to note that the beginning of lung function deterioration is not always accompanied by symptoms, for this reason, serial evaluations are essential ${ }^{6}$. 
A reduction in the values of $\mathrm{FVC}$ and $\mathrm{PEF}$ can be detected even before the first motor manifestations. According to Mayer et al (2018), these parameters suffer an annual drop of 5\%, from 5 to 24 years of age ${ }^{3}$. Advanced stages of Duchenne usually require hospital management. During this period, surgical correction for orthopedic abnormalities may be necessary. In the face of significant reductions in chest wall compliance, especially if FVC declines by more than $40 \%$, the use of non-invasive ventilation (NIV), such as a manual self-inflating bag, is indicated, because of its capacity to contain the loss of FVC to only $0.5 \%$ per year ${ }^{\mathbf{6},{ }^{14} \text {. }}$ In spite of this correlation, there is no high-level evidence to precisely associate the levels of FVC and PEF with the need for airway cleaning devices or mechanical ventilation ${ }^{3}$. Nocturnal ventilation is necessary when PSG studies identify a $\mathrm{SpO} 2$ of $88 \%$ or less, an increase in petCO2 of $10 \mathrm{mmHg}$ above the daytime baseline or petCO2 levels greater than $50 \mathrm{mmHg}$ for more than $2 \%$ of sleep time ${ }^{6}$.

Manually assisted coughing may also be required. It is recommended for patients to have oximeters in their homes and increase the frequency of insufflations whenever $\mathrm{O} 2$ saturation is less than $95 \%$, in order to reduce the recurrence of pneumonia ${ }^{\mathbf{6}, \mathbf{1 5}}$. For the same reason, authors support the use of antibiotics in the presence of early pulmonary infection signs, like fever, hypoxemia, leukocytosis, high C-reactive protein levels, expectoration, or infiltrations on chest X-ray ${ }^{\mathbf{1 6}}$. Brazilian consensus on Duchenne muscular dystrophy (2018) also emphasizes the need for an immunization regimen that includes 3 doses of Pneumococcal Conjugate Vaccine (Pn10 or Pn13), 1 dose of Pneumococcal Polysaccharide Vaccine (Pn23) and annual doses of AntiInfluenza Vaccine in children with DMD, particularly those using corticosteroids ${ }^{\mathbf{1 7}}$.

Steroids, like Prednisone $0.75 \mathrm{mg} / \mathrm{Kg}$ and Prednisolone $0.75 \mathrm{mg} / \mathrm{Kg}$, are first-line drugs used in DMD's therapy, as a second alternative, Deflazacort $0.9-1 \mathrm{mg} / \mathrm{Kg}$ can be prescribed. Meta-analyses showed its potential to increase the life expectancy of the patients by promoting sarcolemmal stabilization, improving motor function, preventing spine deformities, giving longer independent waking, and, mainly slowing down the progression of lung and cardiac injury ${ }^{\mathbf{2}, \mathbf{1 8}}$. Glucocorticoids regimen should be started in boys after 2 years of age who begin to present motor deficits or over 5 years old, regardless of their degree of functionality. Frequently monitoring the possible adverse effects of the medication is crucial, as these patients often use continued corticosteroids throughout life. It is generally recommended that the interval between evaluations does not exceed 6 months, paying attention to cushingoid features and ophthalmological manifestations. Intolerable side effects are one of the few reasons for discontinuing these drugs in DMD ${ }^{2}$.

Antioxidants, like Idebenone, despite not being approved by the Food and Drug Administration, have also proved valuable in maintaining the DMD pulmonary function ${ }^{2}$. A research conducted by McDonald et al (2016) demonstrated a noticeable increase for $\mathrm{FVC} \%$ and a reduction in the incidence of bronchopulmonary adverse events in favor of Idebenone when compared to placebo ${ }^{\mathbf{1 0}}$. Other promising therapies described in the literature are read-through agents (Ataluren), exon skipping agents or even micro-dystrophin gene therapy, such as Drisapersen and Eteplirsen, which, through the deletion of defective exons, allow the production of functional dystrophin or even stem cell transplant, but like Idebenone, require further studies to be consolidated as a treatment for DMD ${ }^{2,19}$.

\section{CONCLUSION}

Duchenne Muscular Dystrophy is an incurable disease with a poor prognosis. Its most severe complication is related to ventilatory muscle failure, for this reason, current disease control measures are focused on maintaining ventilation, preventing infections, and delaying the progression of muscle damage. Early initiation of

patient monitoring, NIV and treatment with steroids are essential to maintain adequate lung function for a longer time and prolong life expectancy.

\section{REFERENCES:}

1. Birnkrant D, Bushby K, Bann C et al. Diagnosis and management of Duchenne muscular dystrophy, part 1: diagnosis, and neuromuscular, rehabilitation, endocrine, and gastrointestinal and nutritional management. The Lancet Neurology . 2018;17(3):251-267. doi:10.1016/s1474-4422(18)30024-3

2. Araujo A, Carvalho A, Cavalcanti E et al. Brazilian consensus on Duchenne muscular dystrophy. Part 
1: diagnosis, steroid therapy and perspectives.Arq Neuropsiquiatr . 2017;75(8):104-113. doi:10.1590/0004$282 \times 20170112$

3. Mayer O. Pulmonary function and clinical correlation in DMD.Paediatr Respir Rev . 2018;30:13-15. doi:10.1016/j.prrv.2018.08.003

4. Aliverti A, LoMauro A, D'Angelo M. Assessment and management of respiratory function in patients with Duchenne muscular dystrophy: current and emerging options.Ther Clin Risk Manag . 2015:1475. doi:10.2147/tcrm.s55889

5. Finder J, Mayer O, Sheehan D et al. Pulmonary Endpoints in Duchenne Muscular Dystrophy. A Workshop Summary.Am J Respir Crit Care Med . 2017;196(4):512-519. doi:10.1164/rccm.201703-0507ws

6. Birnkrant D, Bushby K, Bann C et al. Diagnosis and management of Duchenne muscular dystrophy, part 2: respiratory, cardiac, bone health, and orthopaedic management. The Lancet Neurology . 2018;17(4):347-361. doi:10.1016/s1474-4422(18)30025-5

7. Mayer O, Finkel R, Rummey C et al. Characterization of pulmonary function in Duchenne Muscular Dystrophy.Pediatr Pulmonol . 2015;50(5):487-494. doi:10.1002/ppul.23172

8. Fox H, Millington L, Mahabeer I, van Ruiten H. Duchenne muscular dystrophy.BMJ . 2020:17012. doi:10.1136/bmj.17012

9. Wong S, McClaren B, Archibald A et al. A mixed methods study of age at diagnosis and diagnostic odyssey for Duchenne muscular dystrophy.European Journal of Human Genetics . 2015;23(10):1294-1300. doi:10.1038/ejhg.2014.301

10. McDonald C, Meier T, Voit T et al. Idebenone reduces respiratory complications in patients with Duchenne muscular dystrophy.Neuromuscular Disorders . 2016;26(8):473-480. doi:10.1016/j.nmd.2016.05.008

11. Lo Mauro A, Aliverti A. Physiology of respiratory disturbances in muscular dystrophies.Breathe . 2016;12(4):318-327. doi:10.1183/20734735.012716

12. Buu M. Respiratory complications, management and treatments for neuromuscular disease in children. Curr Opin Pediatr . 2017;29(3):326-333. doi:10.1097/mop.0000000000000498

13. Hoque R. Sleep-Disordered Breathing in Duchenne Muscular Dystrophy: An Assessment of the Literature.Journal of Clinical Sleep Medicine . 2016;12(06):905-911. doi:10.5664/jcsm.5898

14. Katz S, Barrowman N, Monsour A, Su S, Hoey L, McKim D. Long-Term Effects of Lung Volume Recruitment on Maximal Inspiratory Capacity and Vital Capacity in Duchenne Muscular Dystrophy. Ann Am Thorac Soc . 2015. doi:10.1513/annalsats.201507-475bc

15. Toussaint M, Pernet K, Steens M, Haan J, Sheers N. Cough Augmentation in Subjects With Duchenne Muscular Dystrophy: Comparison of Air Stacking via a Resuscitator Bag Versus Mechanical Ventilation.Respir Care . 2015;61(1):61-67. doi:10.4187/respcare.04033

16. Sheehan D, Birnkrant D, Benditt J et al. Respiratory Management of the Patient With Duchenne Muscular Dystrophy.Pediatrics . 2018;142(Supplement 2):S62-S71. doi:10.1542/peds.2018-0333h

17. Araujo A, Nardes F, Fortes C et al. Brazilian consensus on Duchenne muscular dystrophy. Part 2: rehabilitation and systemic care.Arq Neuropsiquiatr . 2018;76(7):481-489. doi:10.1590/0004-282x20180062

18. Matthews E, Brassington R, Kuntzer T, Jichi F, Manzur A. Corticosteroids for the treatment of Duchenne muscular dystrophy. Cochrane Database of Systematic Reviews . 2016. doi:10.1002/14651858.cd003725.pub4

19. Gogou M, Pavlou E, Haidopoulou K. Therapies that are available and under development for Duchenne muscular dystrophy: What about lung function?.Pediatr Pulmonol . 2019;55(2):300-315. doi:10.1002/ppul.24605 